\title{
Ja, nein, vielleicht? Der Einfluss der Partnerschaftsqualität auf die Übereinstimmung der Elternschaftsabsichten von Paaren
}

\author{
Yes, no, maybe? The influence of relationship quality on the \\ correspondence of couples' fertility intentions
}

\begin{abstract}
Zusammenfassung:
In diesem Beitrag stehen die Elternschaftsabsichten von Paaren im Mittelpunkt. Es wird geprüft, ob in Partnerschaften übereinstimmende oder divergierende Elternschaftsabsichten vorliegen. Darüber hinaus wird analysiert, ob ein Zusammenhang zwischen der Partnerschaftsqualität und übereinstimmend positiven Kinderwünschen beider Partner besteht. Ausgangspunkt ist die Annahme, dass eine hohe Partnerschaftsqualität einen positiven Einfluss auf übereinstimmend positive Elternschaftsabsichten hat und eine geringe Partnerschaftsqualität diesen entgegenwirkt. Die Ergebnisse zeigen, dass Paare überwiegend übereinstimmende Intentionen aufweisen. Dennoch äußern 14 Prozent aller untersuchten Paare divergierende Elternschaftsabsichten. Zudem kann erwartungsgemäß ein Zusammenhang zwischen der Partnerschaftsqualität und übereinstimmend positiven Elternschaftsabsichten beobachtet werden.
\end{abstract}

Schlagwörter: partnerschaftlicher Kinderwunsch, Partnerschaftsqualität, kinderlose Paare, Eltern

\begin{abstract}
:
This paper investigates fertility intentions of couples. It is examined to what degree fertility intentions of the respondent and the partner deviate. Furthermore, it is analyzed if the relationship quality relates to couples' fertility intentions. The investigation is based on the assumption that high relationship quality leads to positive fertility intentions of both partners. Vice versa, a low relationship quality results in negative or divergent fertility intentions of both partners. Results show that couples give similar report on their intentions. Only for a minority of 14 percent of the couples, fertility intentions deviate. In accordance with expectations, the multivariate results show that relationship quality and the couples' fertility intentions are closely related.
\end{abstract}

Key words: dyadic fertility intentions, relationship quality, childless couples, parents 


\section{Einführung in die Thematik der dyadischen Kinderwunschforschung}

Kinderwunschanalysen ${ }^{1}$ gehören zu den zentralen Forschungsfeldern der aktuellen Familiendemographie. Bisherige Analysen basieren dabei jedoch fast ausschließlich auf Befragungsdaten, in denen nur die Merkmale einer Person, zumeist der Frauen, berücksichtigt werden (z.B. Engelhardt 2004; Hayford 2009; Heiland/Prskawetz/Sanderson 2008). Diese Betrachtungsweise vernachlässigt, dass generative Entscheidungen (in den meisten Fällen) im Kontext einer Paarbeziehung getroffen und auch umgesetzt werden (Borchardt/ Stöbel-Richter 2004: 39; Huinink/Konietzka 2007: 158; Klaus/Suckow 2005: 104). Die Dispositionen beider Partner sind für die generative Entscheidungsfindung von erheblicher Bedeutung (Pavetic/Stein 2011: 5, 19; Sobel/Arminger 1992: 46). Wird der Kinderwunsch eines Einzelnen ohne den (vielleicht absolut gegenläufigen) Kinderwunsch des aktuellen Partners betrachtet, ist die Aussagekraft des Kinderwunsches als Messinstrument für zukünftige Elternschaft eingeschränkt. Denn in Partnerschaften muss nicht zwingend ein geteilter Kinderwunsch vorliegen (Diefenbach 2005: 118). Es scheint vielmehr ein Aushandlungsprozess zwischen zwei Partnern zu sein, bei dem sich die Partner wechselseitig beeinflussen (Pavetic/Stein 2011: 19). Vor diesem Hintergrund ist die Betrachtung von individuellen Kinderwünschen keinesfalls ausreichend. Dennoch findet bisher kaum eine Differenzierung zwischen individuellen und partnerschaftlichen Kinderwünschen statt (Rosina/Testa 2009: 488), was nicht zuletzt einem Mangel an adäquaten Daten geschuldet ist.

Mit dem Beziehungs- und Familienpanel (pairfam) ist es aufgrund der Erhebung von Daten zu Ankerperson und deren Partner erstmals möglich, den Partnerschaftskontext für Deutschland in den Mittelpunkt von Kinderwunschanalysen zu rücken und zu prüfen, in welchem Umfang individuelle Kinderwünsche innerhalb von Paaren übereinstimmen. Es ist davon auszugehen, dass kongruente Elternschaftsabsichten eine Realisierung wahrscheinlicher werden lassen, da ein gemeinsames „Projekt“ verfolgt wird. Damit könnte ein Hinweis auf zukünftige Geburten gegeben sein. Sollten divergierende Elternschaftsabsichten vorliegen, wäre es denkbar, dass Kinderwünsche aufgeschoben werden (Thomson/Hoem 1998: 319), ein Kompromiss ausgehandelt wird (Testa/Cavalli/Rosina 2011: 158), ein Partner seine Entscheidung in der Zweierbeziehung durchsetzt (Pavetic/Stein 2011: 6) oder aber die Beziehung aufgrund der Uneinigkeit aufgelöst wird (Pavetic/Stein 2011: 19). Die erste Forschungsfrage dieses Beitrags bezieht sich daher auf die Paarebene und welchen Grad der Übereinstimmung Paare hinsichtlich ihrer zeitnahen Elternschaftsabsichten aufweisen. Grundlage für das Konstrukt zeitnaher Elternschaftsabsichten ist dabei die Frage, ob die Befragten in den nächsten zwei Jahren (erneut) Mutter oder Vater werden wollen.

Die Analyse von Fertilitätsprozessen auf Paarebene scheint unabdingbar. Darüber hinaus zeichnet sich ein Zusammenhang zwischen der Partnerschaftsqualität und verschiedenen Fertilitätsprozessen ab (Myers 1997; Rijken/Liefbroer 2009; Rijken/Thomson

1 Der Begriff „Kinderwunsch“ fasst in diesem Papier die verschieden Stufen des Fertilitätsprozesses zusammen. Neben Idealen und Wünschen werden in diesem Papier auch Intentionen und Elternschaftsabsichten unter diesen Oberbegriff subsumiert, auch wenn sie unterschiedliche Stadien des Fertilitätsprozesses abbilden. 
2011). Dies erscheint nur konsequent, da eine bestehende Partnerschaft häufig als Voraussetzung für eine Familiengründung bzw. -erweiterung gesehen wird. Wenn Fertilitätsentscheidungen also im Paarkontext getroffen werden, ist es sehr wahrscheinlich, dass auch die Bewertung dieser Partnerschaft in den Entscheidungsprozess einfließt. Daher soll als zweite Forschungsfrage der Zusammenhang der Partnerschaftsqualität mit übereinstimmend positiven Elternschaftsabsichten auf Paarebene untersucht werden. Grundlage für die Bewertung der Partnerschaftsqualität soll die subjektive Zufriedenheit der Befragten mit ihrer Beziehung insgesamt zum Zeitpunkt der Erhebung sein.

Da die vorliegenden Analysen auf Querschnittsdaten basieren, muss ein grundsätzliches Problem erwähnt werden: Untersuchungen zur kausalen Wirkung der Partnerschaftsqualität auf übereinstimmend positive Elternschaftsabsichten sind erst mit mehreren Erhebungswellen möglich, mit denen dann die zeitliche Ordnung zwischen Partnerschaftsqualität und der Genese des Kinderwunsches analysiert werden kann. Zwar liegen dieser Studie nur Querschnittsdaten zu Grunde, jedoch ist in der Art der Frageformulierung eine gewisse zeitliche Perspektive enthalten. Die Frage zur Beziehungszufriedenheit bezieht sich auf den Zeitpunkt der Erhebung, während sich Aussagen zur Elternschaftsabsicht auf die nächsten zwei Jahre beziehen. So sind erste Aussagen über die Wirkungsweise der Partnerschaftsqualität auf den Kinderwunsch möglich. Längsschnittanalysen müssten die Ergebnisse der vorliegenden Studie jedoch verifizieren.

Die durchgeführten Analysen differenzieren zwischen Eltern und kinderlosen Paaren, da sich diese beiden Gruppen hinsichtlich ihres Erfahrungshorizontes unterscheiden (Arránz Becker 2008: 110ff.; Eckhard/Klein 2006: 79; Heiland et al. 2008: 135). Eltern wissen bereits, welchen Einfluss Kinder auf ihre Paarbeziehung nehmen können, kinderlose Paare hingegen nicht. Das Wissen um die Bedeutung von Elternschaft nimmt auch Einfluss auf die Kinderwunschgenese, weshalb eine Unterscheidung beider Gruppen für die Analyse der genannten Fragestellungen sinnvoll erscheint.

Der vorliegende Beitrag präsentiert zunächst bisherige Forschungsergebnisse zum Einfluss der Partnerschaftsqualität auf den Kinderwunsch bzw. auf die Realisierung von Geburten (Abschnitt 2). Anschließend werden theoretische Überlegungen zur Entstehung von Kinderwünschen dargestellt, die sich auf die soziale Austauschtheorie (Thibaut/Kelley 1959) konzentrieren (Abschnitt 3). Auf dieser Grundlage werden die Hypothesen zum Einfluss der Partnerschaftsqualität auf Elternschaftsabsichten von Paaren formuliert (Abschnitt 3.3). Im darauf folgenden Abschnitt 4 werden die Daten und Methoden präsentiert. Daran schließt sich die Darstellung der multivariaten Ergebnisse an (Abschnitt 5). Der Artikel endet mit einer Diskussion der Ergebnisse (Abschnitt 6).

\section{Forschungsstand zum Thema Kinderwunsch und Partnerschaftsqualität}

Das Defizit an geeignetem Datenmaterial auf Paarebene spiegelt sich auch in der Literatur zur Wirkungsweise der Partnerschaftsqualität auf dyadische Fertilitätsentscheidungen wider. Auf der einen Seite existieren Studien, welche die Übereinstimmung der Partner in den Mittelpunkt stellen (Cavalli/Rosina 2011; Rosina/Testa 2009; Testa 2012; Testa et al. 
2011; Thomson/Hoem 1998) und belegen, dass divergierende Elternschaftsabsichten eher selten sind (Cavalli/Rosina 2011: 28; Rosina/Testa 2009: 492; Testa 2012: 75; Testa et al. 2011: 166; Thomson/Hoem 1998: 315). Zudem weisen Forschungsergebnisse darauf hin, dass die Geburt von (weiteren) Kindern mit verschiedenen Aspekten der Partnerschaftsqualität in Verbindung steht (Rijken/Liefbroer 2009). Demnach weisen insbesondere Frauen mit einer mittelmäßigen Einschätzung ihrer Partnerschaft eine stärkere Neigung zu Erst- und Zweitgeburten auf. Bei Männern hat die Partnerschaftsqualität hingegen kaum einen Einfluss auf den Übergang zum ersten Kind. Der Übergang zum zweiten Kind wird bei ihnen jedoch begünstigt, wenn sie ihre Partnerschaft weder besonders positiv noch besonders negativ bewerten. ${ }^{2}$ Dennoch bleibt offen, ob die Einschätzung der Partnerschaft beider Beziehungspartner Einfluss auf die gemeinsamen Fertilitätsentscheidungen nimmt.

Auf der anderen Seite existieren Studien, welche den Einfluss der Partnerschaftsqualität auf individuelle Kinderwünsche untersuchen. Für Deutschland liegen geschlechtsspezifische Befunde zum Einfluss der Beziehungsstabilität auf den Kinderwunsch vor (Eckhard/Klein 2006; Höhn/Ette/Ruckdeschel 2006; Eckhard/Klein 2007). Nach Analysen von Eckhard und Klein (2006: 67, 179; 2007: 291; 2008: 392) mit dem deutschen Familiensurvey empfinden vor allem Männer die Beziehungsstabilität als Voraussetzung für eine Elternschaft: Werden vom Mann keine Probleme bzw. Konflikte in der Partnerschaft wahrgenommen, zeigt sich ein positiver Effekt auf den Kinderwunsch. Zudem scheinen sich die Elternschaftsabsichten und deren Motivation von Eltern und kinderlosen Paaren zu unterscheiden (Eckhard/Klein 2006: 180ff.; Eckhard/Klein 2007: 285ff.). Bereits erworbene Elternschaftserfahrungen wirken sich auf ,die Wahrnehmung bestimmter Beweg- und Hinderungsgründe" (Eckhard/Klein 2006: 79) aus, was zu einer unterschiedlichen Genese des Kinderwunsches von Eltern und Kinderlosen führen kann. Ein weiteres Indiz für Unterschiede zwischen Eltern und Kinderlosen liefern die Ergebnisse von Höhn et al. (2006: 32, 38) auf Basis der deutschen Stichprobe des Generations and Gender Survey (GGS) 2005, welche die Paarebene über Proxy-Interviews abbildet. Kinderwünsche von Partnern divergieren hier insbesondere dann, wenn es um die Geburt weiterer Kinder geht. Eltern fürchten demnach, dass weitere Kinder ihre Partnerschaftsqualität verringern könnten. Andererseits bewerten Eltern die Gefahr von Partnerschaftskonflikten im Vergleich zu Kinderlosen als weniger gravierend (Klein/Eckhard 2008: 391f.). Analysen auf internationaler Ebene unterstützen die Annahme, dass sich Eltern und Kinderlose unterscheiden. Ergebnisse von Bulatao (1981: 21,23) deuten darauf hin, dass die Geburt eines ersten Kindes größere Konsequenzen für die Partnerschaft hat als Geburten höherer Ordnung. Die Trennungskosten würden beispielsweise mit einem Kind deutlich höher ausfallen als ohne (Lillard/Waite 1993: 656). Neben Veränderungen in den sozialen bzw. familialen Kontakten sowie in der Freizeitgestaltung (Peuckert 1999: 119), nimmt die Zufriedenheit mit der Paarbeziehung nach dem ersten Kind deutlich ab (Gloger-Tippelt/Huerkamp 1998: 633; Jurgan/Gloger-Tippelt/Ruge 1999: 37; Peuckert 1999: 120; Reichle/ Werneck 1999: 12).

Betrachtet man die Studien genauer, in denen die Partnerschaftsqualität als erklärende Variable berücksichtigt wird, fällt auf, dass vor allem deren Operationalisierung stark va-

2 Eine Erweiterung dieser Studie von Rijken und Thomson (2011) bestätigt die genannten Ergebnisse unter Verwendung von Daten zur Partnerschaftsqualität für beide Partner. 
riiert. Während beispielsweise Eckhard und Klein (2006: 68) aufgrund fehlender alternativer Fragestellungen die Beziehungsstabilität anhand jemals wahrgenommener Schwierigkeiten in der Partnerschaft messen, wählen Rijken und Liefbroer (2009: 33f.) ein deutlich differenzierteres Konzept zur Operationalisierung der Partnerschaftsqualität. Sie beziehen positive und negative Interaktionen zwischen den Partnern sowie deren Wertekonsens und mögliche Trennungsabsichten mit ein. Dies verdeutlicht, dass kein Konsens über die ,richtige“ Messung der Partnerschaftsqualität besteht und es teilweise zu starken Abweichungen in Definition und Operationalisierung kommen kann (Xu 1998: 367; Siffert/ Bodenmann 2010: 243). Zudem wurde die Partnerschaftsqualität bisher vorrangig im Rahmen der Ehe evaluiert (z.B. Norton 1983; Thomson/Colella 1992; Lillard/Waite 1993; Myers 1997), was Kohabitation und nichteheliche Partnerschaften ohne gemeinsamen Wohnsitz ausblendet. Diese sind jedoch fester Bestandteil partnerschaftlicher Lebensformen in Deutschland (Klein 2003: 507).

\section{Theoretische Hintergründe und Hypothesen}

\subsection{Abgrenzung verschiedener Kinderwunschkonzepte}

Bisherige Studien zum Thema Kinderwunsch legen verschiedene Kinderwunschkonzepte zugrunde. Die entsprechenden Fragestellungen beziehen unterschiedlich stark persönliche und gesellschaftliche Rahmenbedingungen mit ein und sind daher zum Teil eher abstrakt. Grundsätzlich wird in der Literatur zwischen Wünschen (ideals/desires) und Intentionen (intentions) unterschieden (Hagewen/Morgan 2005: 510; Miller/Pasta 1995: 531, 549; Thomson 2001: 5347). Wünsche können losgelöst von konkreten und persönlichen Realisierungsbedingungen (wie z.B. der Existenz eines Partners) geäußert werden (Thomson 2001: 5348). Intentionen hingegen beziehen neben der individuellen Präferenz auch das persönliche Vermögen mit ein, diese Ziele erreichen zu können (Thomson 2001: 5347). Somit werden bei diesem Konzept auch mögliche individuelle Beschränkungen wie Partnerlosigkeit oder Unfruchtbarkeit berücksichtigt. Insofern sind Intentionen weniger abstrakt als Wünsche. Zudem kann in das Konzept der Intentionen ein zeitlicher Referenzrahmen aufgenommen werden. Dies bietet den Vorteil, dass die Befragten die Realisierungschancen ihrer Kinderwünsche besser einschätzen können und dadurch die Frageformulierung für sie (und ihren Partner) konkreter wird (Ette/Ruckdeschel 2007: 58; van Peer 2002: 118).

Zusammenfassend sind Wünsche und Intentionen dem tatsächlichen Fertilitätsverhalten vorangestellt, wie Abbildung 1 noch einmal darstellt. Wünsche sind weniger konkret als Intentionen und daher diesen vorgelagert (Miller 2010: 2). Für die Analyse von Kinderwünschen im Paarkontext bietet es sich wiederum an, ein Konzept zu verwenden, welches möglichst konkret ist und damit auch die persönlichen Realisierungsbedingungen der Befragten einbezieht. Das in diesem Beitrag verwendete Maß der Elternschaftsabsicht ist den Intentionen zuzuordnen, da hier die persönlichen Rahmenbedingungen einbezogen und z.B. die Existenz eines Partners vorausgesetzt wird. Zudem wird den Befragten ein zeitlicher Referenzrahmen von zwei Jahren vorgegeben. Beide Aspekte grenzen die El- 
ternschaftsabsicht damit deutlich vom Konzept der Wünsche ab. Darüber hinaus sind Intentionen stärker paargeleitet bzw. -orientiert als Wünsche, da diese unabhängig von konkreten und persönlichen Realisierungsbedingungen bestehen können. Partnerschaftsaspekte werden jedoch spätestens mit der konkreten Handlungsabsicht relevant.

Abbildung 1: Verortung der erfragten Kinderwunschkonzepte im Deutschen Beziehungs- und Familienpanel (pairfam)

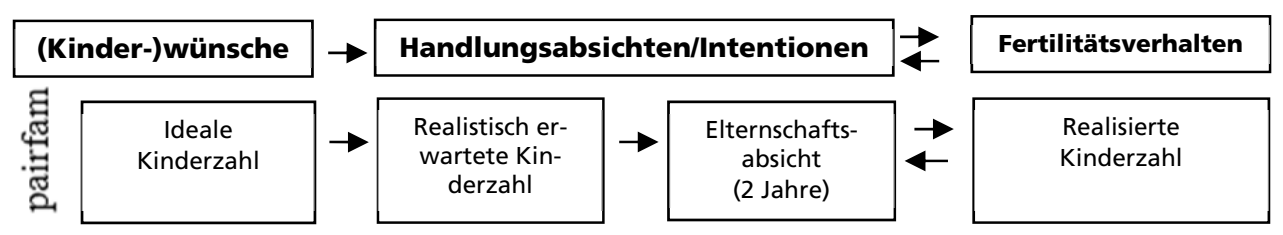

Quelle: Eigene Darstellung.

\subsection{Austauschtheoretischer Hintergrund}

Verschiedene Theorieansätze in der Familienforschung unterstellen Familienbildungsprozessen einen rationalen Entscheidungscharakter, der auf einer Abwägung der KostenNutzen-Aspekte basiert (z.B. theory of planned behavior, austauschtheoretische und familienökonomische Ansätze). Die wenigsten berücksichtigen dabei jedoch eine Interaktion auf Paarebene. Bei der theory of planned behavior (Ajzen 1991) steht das Individuum im Mittelpunkt der Entscheidungsfindung und der Partner wird lediglich über subjektive Annahmen des vom Partner gewünschten Verhaltens berücksichtigt (Ajzen/Klobas 2013: 211). Der familienökonomische Ansatz nach Becker $(1974,1981)$ bietet die Möglichkeit Interaktionen zwischen Partnern über materielle und immaterielle Investitionen auf Haushaltsebene zu integrieren. Dies trifft ebenfalls auf die Austauschtheorie (Thibaut/Kelley 1959) zu, die sich mit Tauschbeziehungen jedweder Art, auseinandersetzt. Da Paarbeziehungen ebenso außerhalb eines gemeinsamen Haushalts bestehen und gemeinsame Kinder damit auch Bestandteil verschiedener Haushalte sein können, erscheint die soziale Austauschtheorie als theoretische Perspektive zur Erklärung des potentiellen Einflusses der Partnerschaftsqualität auf übereinstimmend positive Elternschaftsabsichten am besten geeignet.

Wie alle Rational-Choice-basierten Ansätze stützt sich auch die Austauschtheorie auf rationale Akteure (Klaus/Suckow 2005: 104), welche ihre Handlungsoptionen anhand eines Kosten-Nutzen-Kalküls bewerten und sich an den resultierenden, möglichen Gewinnen orientieren. Ziel ist die Maximierung des individuellen Nutzens durch die gemeinsame Partnerschaft (Hill/Kopp 2002: 107). Elternschaft stellt damit eine mögliche Handlungsoption für ein Paar dar und die damit verbundenen Kosten sowie mögliche Handlungsalternativen werden einer ausführlichen Kosten-Nutzen-Abwägung unterzogen. Übertragen auf die vorliegende Untersuchung der Elternschaftsabsicht fällt die Entscheidung zur Elternschaft für beide Partner dann positiv aus, wenn ein (weiteres) Kind für ein Paar den subjektiv größtmöglichen, gemeinsam zu erzielenden Ertrag im Vergleich zu den verfügbaren Handlungsalternativen verspricht. Dies schließt Austausch- und Aushandlungsprozesse zwischen den Partnern ein, die sich wiederum auch auf die Partnerschaftsqualität auswirken. Neben mate- 
riellen Ressourcen werden auch immaterielle Ressourcen wie Aufmerksamkeit, Verständnis, emotionale Zuwendung oder Hilfe ausgetauscht (Schmidt/Moritz 2009: 35), an denen gerade auf Paarebene kontinuierlich Bedarf besteht (Arránz Becker 2008: 26). Die Partner sind damit auf den Austausch immaterieller Ressourcen angewiesen, um ihr persönliches Nutzenniveau zu steigern.

Beziehungen, in denen beide Partner zufrieden sind, sollten einen höheren Nutzen für die Beteiligten haben und zudem stabilisierend auf die Paarbeziehung wirken (Arránz Becker 2008: 26). Diese Stabilisierung vereinfacht eine weitere Verdichtung der gemeinsamen Partnerschaft. Die Entscheidung für Kinder mit dem aktuellen Partner stellt eine solche Intensivierung der Paarbeziehung dar und wird besonders dann erfolgen, wenn beide Partner sehr zufrieden mit ihrer Beziehung sind. Ist mindestens ein Partner unzufrieden mit der aktuellen Beziehung, erscheint eine Verdichtung der Partnerschaft und damit auch eine gemeinsame, positive Elternschaftsabsicht eher unwahrscheinlich.

\subsection{Hypothesenbildung zu EIternschaftsabsichten auf Paarebene}

Auf Basis dieser theoretischen Überlegungen sowie der Annahme, dass eine hohe Partnerschaftsqualität einen positiven Einfluss auf gemeinsame Kinderwünsche eines Paares hat und eine geringe Partnerschaftsqualität gemeinsamen Kinderwünschen entgegenwirkt, lassen sich verschiedene Forschungshypothesen ableiten.

Vor dem Hintergrund, dass Partnerschaft Interaktion erfordert und der Austausch bzw. die Aushandlung von Kinderwünschen und Elternschaftsabsichten Bestandteil dieser Interaktion sind, ist zu erwarten, dass die meisten Paare übereinstimmende Elternschaftsabsichten aufweisen. Divergierende Absichten sind eher die Ausnahme, da sie wahrscheinlich im Verlauf des Aushandlungsprozesses zwischen den Partnern angepasst werden, um weiterhin ein gemeinsames Ziel als Paar zu verfolgen oder aber einer der Partner setzt sich durch. Hypothese 1 lautet demnach, dass hauptsächlich übereinstimmende Elternschaftsabsichten zwischen zwei Partnern bestehen, unabhängig davon, ob diese positiv oder negativ sind.

Im Kontext einer Paarbeziehung sind die Partner bei der Verfolgung ihres Nutzens interdependent und versuchen durch Interaktion miteinander ihren Nutzen durch die Partnerschaft gemeinsam zu maximieren (Hill/Kopp 2002: 111ff.; Nauck 1989: 52f.; Nauck/Kohlmann 1999: 66; Nave-Herz 2004: 134). Da ein gemeinsames Kind diese wechselseitige Abhängigkeit der Partner und deren Interaktion noch verdichtet, ist zu erwarten, dass ein Kind nur gewünscht wird, wenn die Paarbeziehung und der damit verbundene Austausch materieller und immaterieller Ressourcen bislang als ausreichend zufriedenstellend empfunden wurde (Hill/Kopp 2002: 104; Schmidt/Moritz 2009: 33f.). Demnach kann vermutet werden, dass eine hohe Partnerschaftsqualität (welche die Zufriedenheit mit dem Austausch von Ressourcen widerspiegelt) einen positiven Einfluss auf die gemeinsame Familiengründung bzw. -erweiterung hat. Daher ist zu erwarten, dass eine hohe Partnerschaftsqualität, vermittelt über eine starke subjektive Zufriedenheit mit der Beziehung, mit einer gemeinsamen Intention des Paares einhergeht. Hypothese 2 lautet demnach, dass Paare, die eine hohe Beziehungszufriedenheit aufweisen, eher eine gemeinsame, positive Elternschaftsabsicht angeben als Paare, bei denen mindestens ein Partner unzufrieden ist. 
Die Geburt eines Kindes kann Paaren noch unbekannte Informationen über Kosten und Nutzen einer Elternschaft vermitteln, sodass die Intention für ein weiteres Kind dadurch positiv oder negativ beeinflusst werden könnte (Miller/Pasta 1995; Heiland et al. 2008: 135). Da sich Elternpaare und kinderlose Paare hinsichtlich ihrer Erfahrungswerte, was eine Elternschaft bedeutet, und damit auch hinsichtlich der Kinderwunschgenese deutlich voneinander abgrenzen, könnte man annehmen, dass Eltern die Auswirkungen einer (weiteren) Elternschaft auf ihre Partnerschaft und damit auch auf deren Qualität besser einschätzen können als Kinderlose, welche die Vorstellung einer Elternschaft vielleicht noch stärker idealisieren. Eltern nehmen die Auswirkungen der Geburt weiterer Kinder auf ihre Partnerschaft womöglich bewusster wahr, weshalb die gegenwärtige Partnerschaftsqualität einen stärkeren Einfluss auf (weitere) gemeinsame Intentionen bei Eltern hat. Demnach lautet Hypothese 3, dass ein Einfluss der Partnerschaftsqualität auf gemeinsame, positive Elternschaftsabsichten eher bei Eltern als bei Kinderlosen zu erwarten ist.

\section{Daten und Methode zur Analyse der Elternschaftsabsicht auf Paarebene}

\subsection{Stichprobenselektion}

Für die vorliegenden Analysen wurden die Daten der ersten Welle des Beziehungs- und Familienpanels (pairfam) aus dem Jahr 2008/09 (Release 2.0) verwendet (Huinink/Brüderl/Nauck/Walper/Castiglioni/Feldhaus 2011). Die Verfügbarkeit von Informationen zu Ankerperson und zugehörigem Partner in diesem Datensatz ermöglichen es, umfangreiche Untersuchungen auf Paarebene vorzunehmen.

Die folgenden Analysen beziehen sich auf eine Stichprobe, die auf Daten der Kohorten 1970-74 und 1980-84 der Ankerpersonen beruht, für die ebenfalls Angaben zum Partner vorlagen. Demzufolge sind die Befragten zum Erhebungszeitpunkt 34 bis 38 Jahre (Kohorte 1970-74) bzw. 24 bis 28 Jahre (Kohorte 1980-84) alt. Für die entsprechenden Partner wurde eine untere Altersgrenze von 18 Jahren festgelegt. Darüber hinaus wurde für weibliche Partner eine obere Altersgrenze von 45 Jahren determiniert. Sie orientiert sich an der fertilen Phase der Frau und soll sicherstellen, dass eine geäußerte Elternschaftsabsicht aus biologischer Perspektive in den nächsten Jahren noch umgesetzt werden kann. Darüber hinaus wurden Paare aus den Analysen ausgeschlossen, bei denen zum Zeitpunkt der Befragung eine Schwangerschaft vorlag. Gleiches gilt für Paare, bei denen einer der Partner angegeben hatte, mit hoher Wahrscheinlichkeit nicht in der Lage zu sein auf natürlichem Weg ein Kind zu bekommen bzw. zu zeugen. Lag zudem der angegebene Beziehungsbeginn bei einem der Partner vor dem Alter 14, wurden diese Paare aus Plausibilitätsgründen ebenfalls ausgeschlossen. Des Weiteren wurden nur gegengeschlechtliche Paare in den Analysen berücksichtigt. Die resultierende Stichprobe umfasst also insgesamt 2.126 verheiratete und unverheiratete Paare, die zusätzlich nach ihrem Elternschaftsstatus differenziert wurden (1.476 Elternpaare, 650 kinderlose Paare). 


\subsection{Operationalisierung der Variablen für die multinomialen Regressionen}

\subsubsection{Die abhängige Variable zur Elternschaftsabsicht}

Im Rahmen der ersten Welle des Beziehungs- und Familienpanel (pairfam) wurden im Jahr 2008/09 neben der idealen und realistisch erwarteten Kinderzahl auch zeitnahe Elternschaftsabsichten erfragt: Frauen und Männer, die sich in einer Paarbeziehung befinden, wurden separat befragt, ob sie vorhaben, in den nächsten zwei Jahren Mutter bzw. Vater zu werden. Die entsprechenden Antwortmöglichkeiten sind: ,ja, bestimmt“; ,ja, vielleicht“; „nein, eher nicht“; ,nein, bestimmt nicht"; ,darüber habe ich mir noch keine Gedanken gemacht"; „weiß nicht". Die Frage zur zeitnahen Elternschaftsabsicht wurde jedoch nur an Personen gerichtet, welche die vorhergehende Frage zur realistisch erwarteten (weiteren) Kinderzahl nicht mit „,null“ bzw. ,darüber habe ich mir noch keine Gedanken gemacht“ beantwortet haben.

Für die Generierung der abhängigen Variablen wurden die Antwortkategorien ,ja, bestimmt" und ,ja, vielleicht" als positive Elternschaftsabsichten gewertet. Die Antwortkategorien „nein, eher nicht“ sowie „nein, bestimmt nicht“ wurden als negative Elternschaftsabsichten bewertet. Weist das Paar eine positive und eine negative Intention auf, wird dies als divergierende Elternschaftsabsicht beurteilt. Die Antwortkategorien „darüber habe ich mir noch keine Gedanken gemacht" und „,weiß nicht" gehen nicht in die Zielvariable zur Elternschaftsabsicht auf Paarebene ein. Personen, die realistisch keine Kinder mehr erwarten, werden Personen mit negativen Elternschaftsabsicht gleichgesetzt und als solche in die Analysen aufgenommen. Es gibt Argumente dafür, diese Gruppe nicht in die abhängige Variable zu integrieren, da Personen, die sich - womöglich unabhängig - von ihrer Partnerschaftsqualität keine Kinder mehr wünschen, zu Verzerrungen in den Ergebnissen führen können. Allerdings erscheinen gerade Paare ohne weitere Elternschaftsabsicht - vor allem bei den Kinderlosen - als besonders relevant. ${ }^{3}$

Tabelle 1 dokumentiert die Übereinstimmung der Elternschaftsabsicht von Eltern und kinderlosen Paaren. Dabei zeigt sich, dass sowohl Kinderlose als auch Eltern zum großen Teil kongruente Elternschaftsabsichten (beide ja: 28 Prozent, beide nein: 58 Prozent) aufweisen. Nur ein geringer Prozentsatz der Paare ist sich uneins über die Absicht in den nächsten zwei Jahren Mutter oder Vater zu werden (divergierend: 14 Prozent), was den Ausführungen von Hypothese 1 entspricht. Zudem zeigen sich Unterschiede zwischen Eltern und kinderlosen Paaren. Während sich Elternpaare häufiger einig darüber sind, dass sie in den nächsten 2 Jahren kein Kind bekommen möchten (Eltern: 68 Prozent, Kinderlose: 37 Prozent), sind sich kinderlose Paare häufiger einig, ein Kind haben zu wollen (Kinderlose: 43 Prozent, Eltern: 21 Prozent) bzw. haben häufiger unterschiedliche Elternschaftsabsichten (Kinderlose: 20 Prozent, Eltern: 11 Prozent). Betrachtet man die divergierenden Elternschaftsabsichten nach Geschlecht, werden kaum Unterschiede deutlich: Weder die Frauen noch die Männer sagen auffällig häufiger ,ja“, während der Partner anderer Meinung ist (Mann ja/Frau nein: 47 Prozent, Frau ja/Mann nein: 53 Prozent).

3 Um zu prüfen, ob andere Faktoren für die nicht vorhandene Elternschaftsentscheidung verantwortlich sind, wird in den Modellen z.B. für die Anzahl der vorhandenen Kinder kontrolliert. Weiterhin werden zur Überprüfung im Folgenden auch Modelle geschätzt, welche die Personengruppe, die realistisch gesehen keine Kinder mehr erwartet, ausschließen (siehe verwendete Methoden unter 4.3). 
Tabelle 1: Verteilung der Elternschaftsabsicht (EA) auf Paarebene nach Elternschaftsstatus

\begin{tabular}{lcccccc}
\hline Elternschaftsabsicht (EA) auf Paarebene & \multicolumn{2}{c}{ Elternpaare } & \multicolumn{2}{c}{ Kinderlose Paare } & \multicolumn{2}{c}{ Total } \\
\hline & Fälle & Prozent & Fälle & Prozent & Fälle & Prozent \\
Beide haben positive EA & 315 & 21 & 277 & 43 & 592 & 28 \\
Beide haben negative EA & 1.001 & 68 & 240 & 37 & 1.241 & 58 \\
Beide haben divergierende EA & 160 & 11 & 133 & 20 & 293 & 14 \\
\hline Total & 1.476 & 100 & 650 & 100 & 2.126 & 100 \\
\hline
\end{tabular}

Datenbasis: pairfam 2008/09, Release 2.0, eigene Berechnungen, ungewichtete Daten

\subsubsection{Die unabhängige Variable zur Beziehungszufriedenheit}

Nach Hahlweg und Baucom (2008: 3) existieren zur Bewertung der Partnerschaftsqualität keine objektiven Kriterien. Zudem besteht kein theoretisches Fundament, welches Aufnahme oder Ausschluss einzelner Beziehungsaspekte begründet (Arránz Becker 2008: 15). Besonders wesentlich erscheint das Argument, dass sich einzelne Aspekte der Partnerschaftsqualität (z.B. Paarinteraktion und Konfliktverhalten) überschneiden und daher existierende Skalen zur Partnerschaftsqualität nicht geeignet sind, diese adäquat abzubilden (Arránz Becker 2008: 15ff.). Als Konsequenz fehlender objektiver Kriterien wird daher für die vorliegenden Analysen die subjektive Beziehungszufriedenheit als Indikator für die Partnerschaftsqualität herangezogen. Die Partner geben, jeweils getrennt voneinander an, wie zufrieden sie insgesamt mit ihrer Beziehung sind. Dies ist ein eher globales Maß der Wahrnehmung einer Beziehung (Arránz Becker 2008: 17) und schließt alle denkbaren Beziehungsbereiche mit ein, die dem Befragten subjektiv wichtig erscheinen. Damit wird die Interaktion der Partner ebenso berücksichtigt, wie deren Zufriedenheit mit der Aufgabenteilung im Haushalt oder auch der Sexualität. Dieser Beitrag geht davon aus, dass eine hohe subjektive Beziehungszufriedenheit der Befragten mit einer Bündelung positiver Beziehungsaspekte gleichgesetzt werden kann. Wohingegen eine eher geringe Beziehungszufriedenheit mit einer Bündelung negativer Beziehungsaspekte assoziiert wird. Dies gibt indirekt Aufschluss über die Beziehungsstabilität und ermöglicht so eine umfassende Messung des Einflusses der Partnerschaftsqualität auf die Elternschaftsabsichten von Paaren.

Die subjektive Beziehungszufriedenheit wird im Beziehungs- und Familienpanel über die Frage „Wie zufrieden sind Sie insgesamt mit Ihrer Beziehung?“ erhoben. Diese kann mit Hilfe einer 11-stufigen Skala beantwortet werden, die von „sehr unzufrieden“ (Skalenwert 0) bis „sehr zufrieden“ (Skalenwert 10) reicht. Darüber hinaus gibt es die Antwortmöglichkeiten „,weiß nicht“ und „das möchte ich nicht beantworten“, die in der Zielvariablen nicht berücksichtigt werden. Aus der genannten Skala wurde eine kategoriale Variable auf Paarebene erstellt: „,beide zufrieden/sehr zufrieden“ (Skalenwert 7 bis 10) sowie ,,mindestens einer unzufrieden" (mindestens ein Partner gibt einen Skalenwert 0 bis 6 an). Eine differenziertere Einteilung nach „beide zufrieden“, „beide unzufrieden“ und „divergierende Beziehungszufriedenheit" war aufgrund zu geringer Fallzahlen für Eltern und Kinderlose in der Kategorie „beide unzufrieden“ nicht möglich. Dies ergibt sich daraus, dass Paare grundsätzlich eine recht hohe subjektive Zufriedenheit mit ihrer Beziehung angeben (Mittelwert: 8,2). Möglicherweise liegt hier eine positive Selektivität der Stichprobe zugrunde, die sich daraus ergibt, dass nur diejenigen Paare zusammen bleiben, welche die Zufriedenheit mit ih- 
rer Beziehung als überdurchschnittlich beurteilen. Personen, die unzufrieden sind, trennen sich hingegen.

\subsubsection{Weitere Kontrollvariablen}

Neben der übereinstimmenden Elternschaftsabsicht und der subjektiven Beziehungszufriedenheit gehen weitere Kontrollvariablen in die Modelle ein. Zum einen werden für Eltern und Kinderlose Kontrollvariablen für die Altersdifferenz zwischen den Partnern, dem Alter der Partnerin, die Erwerbskonstellation sowie Herkunft, Bildung, Religionszugehörigkeit und Beziehungsdauer der Paare generiert. Zum anderen wird für Elternpaare zusätzlich jeweils eine Kontrollvariable zur Parität auf Paarebene und zur Überprüfung, ob einer der Partner noch kinderlos ist, erstellt.

Die Kontrollvariablen zum Alter beziehen sich auf die Differenz des Lebensalters beider Partner. Im Schnitt beträgt diese Differenz etwa drei Jahre, um die der Mann älter ist als seine Partnerin (Klein 1996: 346). Daran orientiert sich die vorliegende Kategorisierung: Der Mann ist mehr als vier Jahre älter als seine Partnerin, die Frau ist mehr als ein Jahr älter als ihr Partner; Referenz: Alle Paare, bei denen die Frau maximal ein Jahr älter als ihr Partner oder der Mann maximal vier Jahre älter als seine Partnerin ist. Ergänzend dazu wird für das Alter der Partnerin kontrolliert, da die reproduktive Phase der Frau die Paarfertilität begrenzt: Alter der Frau bis 29 Jahre, 30 bis 34 Jahre (Referenz), 35+ Jahre.

Die Einteilung in die Kontrollvariable zur Herkunft basiert auf den Angaben der Befragten zu ihrer Geburtsregion bzw. ihrem Geburtsland: West-West-Partnerschaft (Referenz), Ost-Ost-Partnerschaft, Ost-West-Partnerschaft, mindestens ein Partner wurde im Ausland geboren.

Für die Einordnung in die Strukturvariable zur Bildung wird der jeweils höchste Schulabschluss der Partner zugrunde gelegt. Die Angaben zur Bildung wurden in folgende vier Kategorien eingeteilt, die sowohl Differenzen als auch Übereinstimmungen der Partner hinsichtlich ihrer Bildung erfassen sollen: Der Mann weist einen höheren Bildungsabschluss (hoch/mittel) auf als seine Partnerin (mittel/niedrig) (Referenz), die Frau hat einen höheren Bildungsabschluss (hoch/mittel) als ihr Partner (mittel/niedrig), beide weisen einen mittleren/niedrigen Schulabschluss auf (kein Abschluss, Hauptschule, POS 8./9. Klasse, anderer Abschluss, Realschule, POS 10. Klasse), beide Partner haben einen hohen Abschluss (Fachhochschulreife/Abitur). Liegt für einen Partner keine Angabe zum Schulabschluss vor, wird das Paar nicht in den Analysen berücksichtigt.

Die Strukturvariable zur Erwerbskonstellation basiert auf den Angaben der befragten Paare zu ihrer Teilzeit- bzw. Vollzeiterwerbstätigkeit: Beide Partner erwerbstätig (Zweiverdiener-Modell, Referenz), nur der Mann erwerbstätig (Einverdiener-Modell), Sonstige (nur die Frau erwerbstätig, keiner von beiden erwerbstätig). Liegen keine Angaben zur Erwerbssituation beider Partner vor oder ist ein Partner noch in einer Ausbildung, werden fehlende Werte kodiert.

Mit zunehmender Dauer der Beziehung wird von einer verstärkten Institutionalisierung der Partnerschaft ausgegangen, die einen Kinderwunsch unterstützt. Es wurden folgende Kategorien erstellt: Bis zu zwei Jahre, drei bis vier Jahre, fünf bis sechs Jahre (Referenz), sieben bis acht Jahre, neun bis zehn Jahre, elf und mehr Jahre. 
Die Kontrollvariable zur Religion basiert auf der Frage, ob die einzelnen Personen einer Religionsgemeinschaft angehören, differenziert aber nicht zwischen den einzelnen Konfessionen. Daraus ergeben sich folgende Kategorien: Beide Partner gehören einer Religionsgemeinschaft an (Referenz), kein Partner gehört einer Religionsgemeinschaft an, nur einer der Partner gehört einer Religionsgemeinschaft an. Liegen keine Informationen zur Religionszugehörigkeit vor, werden fehlende Werte kodiert.

Die Differenzierung in Eltern und Kinderlose basiert auf den Angaben der Befragten zu ihren Kindern. Hat mindestens ein Partner ein leibliches Kind, wird das Paar in den vorliegenden Analysen als Elternpaar gewertet. Sind beide Partner noch kinderlos, werden sie den kinderlosen Paaren zugewiesen.

Für Eltern wird zusätzlich eine Kontrollvariable zur Parität auf Paarebene generiert. Dabei wird berücksichtigt, ob die angegebenen, leiblichen Kinder gemeinsame Kinder der Partner sind oder nicht. Es ergeben sich folgende Kategorien: Parität 1 (Referenz) bedeutet, die Partner haben ein gemeinsames Kind oder einer der Partner hat ein Kind mit einer anderen Person. Parität 2+ orientiert sich am gesellschaftlichen Ideal von zwei Kindern, mit deren Erreichen für die meisten Paare die Familienplanung als beendet gilt. Diese Kategorie wird Paaren zugewiesen, die zwei (oder mehr) gemeinsame Kinder haben oder die beide jeweils (mindestens) ein leibliches Kind mit einer Person haben, die nicht ihr aktueller Partner ist. Diese Kategorie trifft ebenfalls auf Paare zu, in denen einer der Partner (mindestens) zwei Kinder mit einer oder mehreren anderen Personen hat. Zusätzlich ist es möglich, dass die Partner ein (oder mehr) gemeinsame(s) Kind(er) haben und gleichzeitig ein Partner ein leibliches Kind mit einer weiteren Person hat.

Außerdem wurde für Elternpaare eine Kontrollvariable aufgenommen, die prüft, ob einer der Partner noch kinderlos ist. Es wird angenommen, dass die bestehende Kinderlosigkeit eines Partners Einfluss auf die gemeinsamen Elternschaftsabsichten nehmen könnte.

Tabelle 2 zeigt die Deskription der Stichprobe, die darstellt, dass eine hohe Beziehungszufriedenheit bei Eltern wie Kinderlosen mit einer übereinstimmend positiven Elternschaftsabsicht zu korrelieren scheint. Allerdings deutet sich auch ein Zusammenhang zwischen einer hohen Zufriedenheit mit der Beziehung und einer einstimmig nicht vorhandenen Elternschaftsabsicht an. Für Eltern könnte sich dies über die bereits erreichte Kinderzahl erklären lassen. Kinderlose könnten in ihrer Lebensplanung die Familiengründung erst zu einem späteren Zeitpunkt geplant haben oder Kinder gar nicht in dieser vorsehen. 
Tabelle 2: Deskription der Stichprobe zu übereinstimmenden Elternschaftsabsichten (EA) auf Paarebene (inklusive der Paare, die beide kein Kind mehr erwarten), Spaltenprozente

\begin{tabular}{|c|c|c|c|c|c|c|c|c|}
\hline & \multicolumn{4}{|c|}{ Elternpaare } & \multicolumn{4}{|c|}{ Kinderlose Paare } \\
\hline & $\begin{array}{c}\text { Positive } \\
\text { EA } \\
N=315\end{array}$ & $\begin{array}{c}\text { Nega- } \\
\text { tive EA } \\
\mathrm{N}=1.001\end{array}$ & $\begin{array}{c}\text { Diverg. } \\
\text { EA } \\
N=160\end{array}$ & $\begin{array}{l}\text { Gesamt } \\
\mathrm{N}=1.476\end{array}$ & $\begin{array}{l}\text { Positive } \\
\text { EA } \\
N=277\end{array}$ & $\begin{array}{c}\text { Nega- } \\
\text { tive EA } \\
\mathrm{N}=240\end{array}$ & $\begin{array}{c}\text { Diverg. } \\
\text { EA } \\
N=133\end{array}$ & $\begin{array}{l}\text { Gesan } \\
\mathrm{N}=65\end{array}$ \\
\hline \multicolumn{9}{|l|}{ Beziehungszufriedenheit der Partner } \\
\hline beide (sehr) zufrieden (RG) & 85 & 76 & 76 & 78 & 88 & 82 & 83 & 85 \\
\hline mindestens ein Partner ist unzufrieden & 15 & 24 & 24 & 22 & 12 & 18 & 17 & 15 \\
\hline \multicolumn{9}{|l|}{ Altersdifferenz zwischen den Partnern } \\
\hline Männer bis 4 Jahre älter als Frau (RG) & 50 & 54 & 44 & 52 & 53 & 59 & 54 & 56 \\
\hline Frau 1+ Jahre älter als Partner & 10 & 11 & 11 & 11 & 7 & 9 & 8 & 8 \\
\hline Mann 4+ Jahre älter als Partnerin & 40 & 35 & 44 & 37 & 40 & 32 & 38 & 36 \\
\hline \multicolumn{9}{|l|}{ Alter der Partnerin } \\
\hline unter 30 Jahre & 55 & 17 & 46 & 28 & 79 & 84 & 90 & 83 \\
\hline 30 bis 34 Jahre (RG) & 15 & 14 & 15 & 14 & 8 & 3 & 3 & 5 \\
\hline $35+$ Jahre & 30 & 69 & 39 & 57 & 13 & 13 & 7 & 12 \\
\hline \multicolumn{9}{|l|}{ Herkunft der Partner } \\
\hline West-West-Paar (RG) & 51 & 58 & 57 & 57 & 59 & 74 & 63 & 66 \\
\hline Ost-Ost-Paar & 17 & 21 & 13 & 19 & 21 & 11 & 13 & 16 \\
\hline Ost-West-Paar & 8 & 5 & 6 & 6 & 7 & 5 & 10 & 7 \\
\hline mind. ein Partner aus dem Ausland & 24 & 16 & 24 & 19 & 13 & 10 & 14 & 12 \\
\hline \multicolumn{9}{|l|}{ Bildungskonstellation des Paares } \\
\hline Mann höhere Bildung als Frau (RG) & 47 & 56 & 43 & 53 & 14 & 14 & 17 & 15 \\
\hline Frau höhere Bildung als Mann & 42 & 35 & 38 & 37 & 26 & 28 & 20 & 25 \\
\hline beide niedrigere/mittlere Bildung & 8 & 5 & 14 & 7 & 21 & 15 & 20 & 18 \\
\hline beide hohe Bildung & 3 & 3 & 5 & 3 & 40 & 44 & 43 & 42 \\
\hline \multicolumn{9}{|l|}{ Erwerbskonstellation des Paares } \\
\hline beide erwerbstätig (RG) & 47 & 56 & 43 & 53 & 78 & 50 & 69 & 66 \\
\hline nur Mann erwerbstätig & 42 & 35 & 38 & 37 & 10 & 15 & 8 & 12 \\
\hline sonstige (nur Frau, keiner erwerbstätig) & 8 & 5 & 14 & 7 & 7 & 20 & 13 & 13 \\
\hline missings & 3 & 3 & 5 & 3 & 4 & 14 & 11 & 9 \\
\hline \multicolumn{9}{|l|}{ Dauer der Paarbeziehung } \\
\hline bis 2 Jahre & 7 & 3 & 8 & 5 & 15 & 25 & 26 & 21 \\
\hline 3-4 Jahre & 19 & 5 & 11 & 9 & 23 & 23 & 20 & 22 \\
\hline 5-6 Jahre (RG) & 18 & 8 & 14 & 11 & 20 & 20 & 23 & 21 \\
\hline 7-8 Jahre & 15 & 10 & 19 & 12 & 17 & 16 & 14 & 16 \\
\hline 9-10 Jahre & 15 & 12 & 14 & 13 & 14 & 6 & 10 & 10 \\
\hline 11+ Jahre & 25 & 62 & 33 & 51 & 12 & 10 & 7 & 10 \\
\hline \multicolumn{9}{|l|}{$\begin{array}{l}\text { Religionszugehörigkeit der Partner } \\
\text { beide gehören Religionsgemeinschaft }\end{array}$} \\
\hline an $(R G)$ & 59 & 56 & 56 & 57 & 51 & 57 & 60 & 55 \\
\hline keiner gehört Religionsgemeinschaft an & 18 & 22 & 15 & 20 & 20 & 13 & 15 & 16 \\
\hline Nur einer gehört Religionsgemeinschaft an & 20 & 20 & 25 & 20 & 25 & 29 & 22 & 26 \\
\hline missings & 3 & 2 & 4 & 3 & 4 & 2 & 3 & 3 \\
\hline \multicolumn{9}{|l|}{ Kinderlosigkeit eines Partners } \\
\hline beide Eltern (RG) & 85 & 93 & 87 & 90 & . & . & . & \\
\hline einer noch kinderlos & 15 & 7 & 13 & 10 & . & . & . & \\
\hline
\end{tabular}




\begin{tabular}{|c|c|c|c|c|c|c|c|c|}
\hline & \multicolumn{4}{|c|}{ Elternpaare } & \multicolumn{4}{|c|}{ Kinderlose Paare } \\
\hline & $\begin{array}{c}\text { Positive } \\
\text { EA } \\
N=315\end{array}$ & $\begin{array}{c}\text { Nega- } \\
\text { tive EA } \\
\mathrm{N}=1.001\end{array}$ & $\begin{array}{c}\text { Diverg. } \\
E A \\
N=160\end{array}$ & $\begin{array}{l}\text { Gesamt } \\
N=1.476\end{array}$ & $\begin{array}{l}\text { Positive } \\
\text { EA } \\
\mathrm{N}=277\end{array}$ & $\begin{array}{c}\text { Nega- } \\
\text { tive EA } \\
\mathrm{N}=240\end{array}$ & $\begin{array}{c}\text { Diverg. } \\
E A \\
N=133\end{array}$ & $\begin{array}{l}\text { Gesamt } \\
N=650\end{array}$ \\
\hline \multicolumn{9}{|c|}{ Kinderzahl auf Paarebene } \\
\hline 1 Kind (RG) & 73 & 19 & 54 & 34 & . & & . & . \\
\hline $2+$ Kinder & 27 & 81 & 46 & 66 & & & & \\
\hline
\end{tabular}

Datenbasis: pairfam 2008/09, Release 2.0, eigene Berechnungen, ungewichtete Daten, EA = Elternschaftsabsichten, $\mathrm{RG}=$ Referenzgruppe

\subsection{Methoden}

Die multivariaten Analysen des vorliegenden Beitrags basieren auf multinomialen logistischen Regressionsmodellen. In diesen werden insgesamt drei unterschiedliche Ausprägungen der Übereinstimmung von Elternschaftsabsichten betrachtet: (1) beide Partner wollen in den nächsten zwei Jahren Mutter und Vater werden (Referenz), (2) das Paar stimmt darin überein, nicht Mutter und Vater werden zu wollen sowie (3) die Partner haben divergierende Elternschaftsabsichten für die nächsten zwei Jahre.

Es werden jeweils getrennte Modelle für Eltern und Kinderlose betrachtet (siehe Ergebnisse Modelle 1 und 2). In einem zweiten Schritt werden Paare, die keine Kinder mehr erwarten, aus den Analysen ausgeschlossen, so dass geprüft werden kann, ob die Beziehungszufriedenheit einen anderen Einfluss auf diese Gruppe nimmt (siehe Ergebnisse Modelle 3 und 4). Um den Forschungsfragen auf Paarebene gerecht zu werden, wurde nicht nur die abhängige Variable auf Paarebene generiert, sondern ebenfalls alle Kontrollvariablen (siehe Operationalisierung unter 4.2.3). ${ }^{4}$

Damit evaluiert werden kann, ob sich Eltern und Kinderlose tatsächlich hinsichtlich des Einflusses der Beziehungszufriedenheit auf die Übereinstimmung der Elternschaftsabsichten unterscheiden, werden Interaktionen zwischen dem Elternschaftsstatus und der Beziehungszufriedenheit in einem Modell berücksichtigt, in das sowohl Eltern als auch Kinderlose integriert sind (siehe Ergebnisse Modelle 5 und 6).

\section{Ergebnisse der multinomialen logistischen Regressionen}

Die Ergebnisse der multinomialen Regressionen werden im Folgenden entsprechend der Modelle separat für Elternpaare und Kinderlose präsentiert. In den Modellen werden jeweils die „Relative Risk Ratios“ ausgewiesen; zur besseren Lesbarkeit des Textes werden diese im Folgenden als „Chance“ bezeichnet.

4 In den Modellen wird zudem für fehlende Werte in den unabhängigen Kontrollvariablen kontrolliert. Aus den zentralen unabhängigen Variablen zur Partnerschaftsqualität werden die missings jedoch ausgeschlossen. 


\subsection{Einfluss der Beziehungszufriedenheit auf die Elternschaftsabsichten von Paaren}

Die Modelle 1 und 2 (Tabelle 3) zeigen die Ergebnisse der Regressionsmodelle für Elternpaare, mit (Modell 1) und ohne Personen, die realistisch keine Kinder mehr erwarten (Modell 2). Für Elternpaare wird in Modell 1 ein signifikanter Effekt der Beziehungszufriedenheit auf Elternschaftsabsichten festgestellt: Die Chance, dass Paare negative oder divergierende Elternschaftsabsichten im Vergleich zur Referenz (positive Elternschaftsabsichten) haben, ist deutlich erhöht, wenn im Vergleich mindestens ein Partner unzufrieden mit der Beziehung ist. Dies bestätigt Hypothese 2 zur positiven Korrelation zwischen einer hohen Beziehungszufriedenheit und einer gemeinsamen Elternschaftsabsicht beider Partner. Dieser Zusammenhang ist höchst bzw. hoch signifikant. Schließt man die Paare aus den Analysen aus, die keine Kinder mehr erwarten, verstärkt sich dieser Effekt noch (Modell 2).

Die Modelle 3 und 4 stellen die Ergebnisse der Regressionsmodelle für kinderlose Paare dar (Tabelle 3). Unter Berücksichtigung der Kontrollvariablen zeigt sich in Modell 3 folgender Effekt der Beziehungszufriedenheit: Ist mindestens ein Partner unzufrieden mit der aktuellen Beziehung, ist die Chance erhöht, dass beide Partner negative bzw. divergierende Elternschaftsabsichten haben. Diese Effekte sind schwach bzw. nicht signifikant, weshalb hier von Tendenzen gesprochen werden kann. Schließt man die Paare, die keine Kinder mehr erwarten, aus den Analysen aus, wird der Effekt geringfügig kleiner (Modell 4). Zusammenfassend kann Hypothese 2 zur Korrelation zwischen einer hohen Beziehungszufriedenheit und gemeinsamer Elternschaftsabsichten für Eltern und für Kinderlose (zumindest teilweise) angenommen werden. 
Tabelle 3: Ergebisse der multinominalen Regression zu übereinstimmenden Elternschaftsabsichten (EA) auf Paarebene für Eltern und kinderlose Paare, relative risk ratios

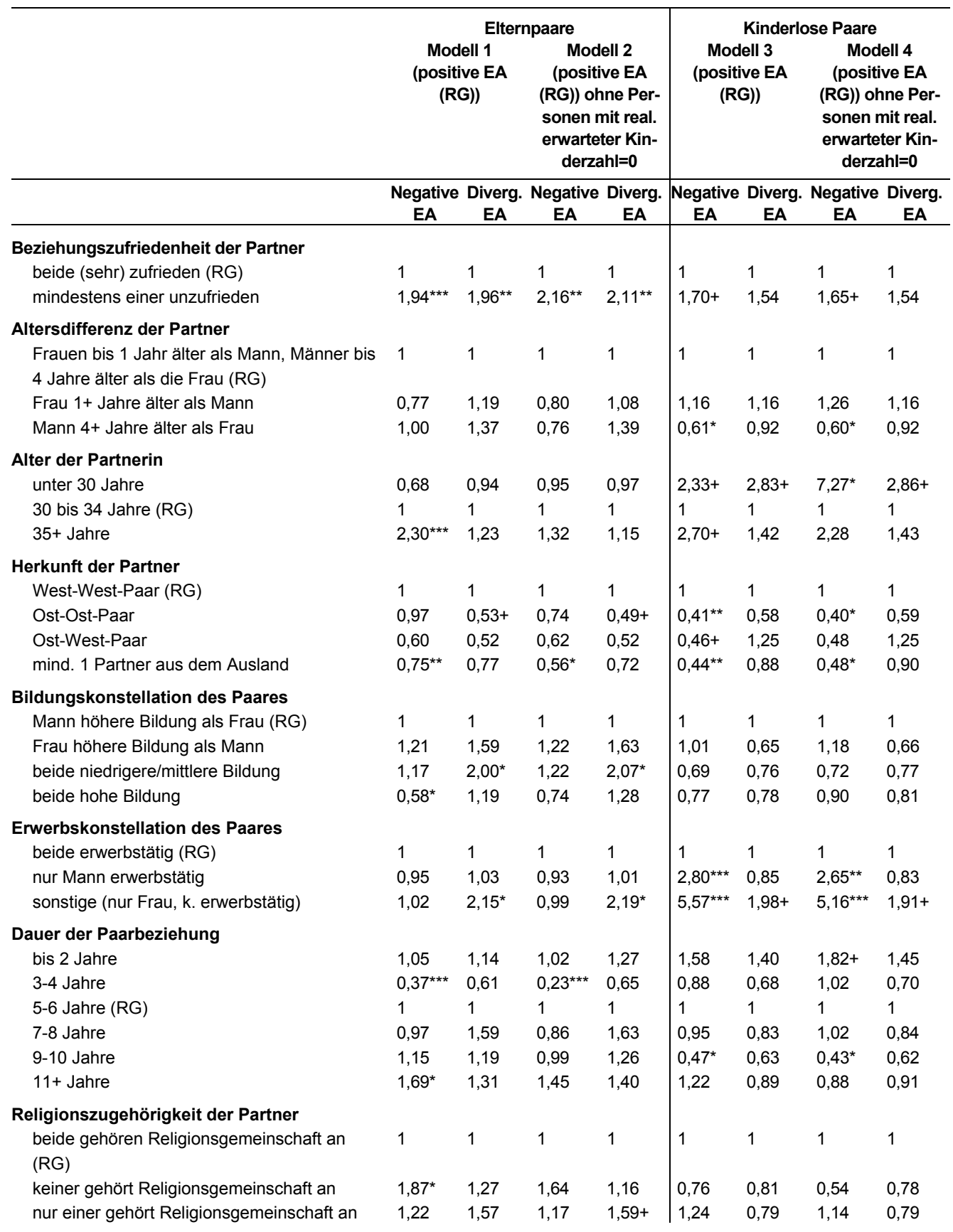




\begin{tabular}{|c|c|c|c|c|c|c|c|c|}
\hline & \multicolumn{4}{|c|}{ Elternpaare } & \multicolumn{4}{|c|}{ Kinderlose Paare } \\
\hline & \multicolumn{2}{|c|}{$\begin{array}{c}\text { Modell } 1 \\
\text { (positive EA } \\
\text { (RG)) }\end{array}$} & \multicolumn{2}{|c|}{$\begin{array}{c}\text { Modell } 2 \\
\text { (positive EA } \\
\text { (RG)) ohne Per- } \\
\text { sonen mit real. } \\
\text { erwarteter Kin- } \\
\text { derzahl=0 }\end{array}$} & \multicolumn{2}{|c|}{$\begin{array}{c}\text { Modell } 3 \\
\text { (positive EA } \\
\text { (RG)) }\end{array}$} & \multicolumn{2}{|c|}{$\begin{array}{c}\text { Modell } 4 \\
\text { (positive EA } \\
\text { (RG)) ohne Per- } \\
\text { sonen mit real. } \\
\text { erwarteter Kin- } \\
\text { derzahl=0 }\end{array}$} \\
\hline & $\begin{array}{c}\text { Negative } \\
\text { EA }\end{array}$ & $\begin{array}{l}\text { Diverg. } \\
\text { EA }\end{array}$ & $\begin{array}{c}\text { Negative } \\
\text { EA }\end{array}$ & $\begin{array}{c}\text { Diverg. } \\
\text { EA }\end{array}$ & $\begin{array}{c}\text { Negative } \\
\text { EA }\end{array}$ & $\begin{array}{c}\text { Diverg. } \\
\text { EA }\end{array}$ & $\begin{array}{c}\text { Negative } \\
\text { EA }\end{array}$ & $\begin{array}{c}\text { Diverg. } \\
\text { EA }\end{array}$ \\
\hline \multicolumn{9}{|l|}{ Kinderlosigkeit eines Partners } \\
\hline beide Eltern (RG) & 1 & 1 & 1 & 1 & . & . & . & . \\
\hline mind. einer noch kinderlos & 0,90 & 0,89 & 1,13 & 0,83 & . & . & . & . \\
\hline \multicolumn{9}{|l|}{ Parität auf Paarebene } \\
\hline 1 Kind (RG) & 1 & 1 & 1 & 1 & . & . & . & . \\
\hline $2+$ Kinder & $9,01^{* * *}$ & $2,10^{* * *}$ & $8,54^{* * *}$ & $2,22^{\star *}$ & . & . & . & . \\
\hline $\mathbf{N}$ & \multicolumn{2}{|c|}{1.476} & \multicolumn{2}{|c|}{776} & \multicolumn{2}{|c|}{650} & \multicolumn{2}{|c|}{614} \\
\hline Pseudo $\mathbf{R}^{2}$ & \multicolumn{2}{|c|}{0,2201} & \multicolumn{2}{|c|}{0,1544} & \multicolumn{2}{|c|}{0,0892} & \multicolumn{2}{|c|}{0,1096} \\
\hline
\end{tabular}

$* * * \mathrm{p}<=0,001$ (höchst signifikant), $* * \mathrm{p}<=0,01$ (hoch sign.), $* \mathrm{p}<=0,05$ (sign.), $+\mathrm{p}<=0,1$ (schwach sign.)

Datenbasis: pairfam 2008/09, Release 2.0, eigene Berechnungen, ungewichtete Daten, kontrolliert für fehlende Werte in den Kontrollvariablen, EA = Elternschaftsabsichten, $\mathrm{RG}=$ Referenzgruppe

\subsection{Einfluss der Kontrollvariablen auf die Elternschaftsabsichten von Paaren}

Neben der Beziehungszufriedenheit weisen auch verschiedene Kontrollvariablen Zusammenhänge zur Übereinstimmung der Elternschaftsabsichten auf Paarebene auf. Die folgende Beschreibung konzentriert sich auf die signifikanten Ergebnisse.

Bei den Elternpaaren (Modell 1) zeigt sich für das Alter der Partnerin, dass negative Elternschaftsabsichten häufiger geäußert werden, wenn die Frau 35 Jahre und älter ist. Weist mindestens ein Partner eine ausländische Herkunft auf, werden seltener negative Elternschaftsabsichten auf Paarebene genannt. Wurden beide Partner in Ostdeutschland geboren, werden zudem seltener divergierende Intentionen angegeben.

Eine hohe Bildung beider Partner verringert die Chance deutlich, dass beide Partner negative Elternschaftsabsichten für die nächsten zwei Jahre formulieren. Gleichzeitig ist die Chance für Paare mit niedrigem/mittlerem Bildungsniveau erhöht, dass divergierende Intentionen vorliegen. Die Erwerbskonstellation nimmt Einfluss, wenn nur die Frau bzw. keiner von beiden erwerbstätig ist. Dann ist die Chance erhöht, dass beide Partner divergierende Elternschaftsabsichten äußern. Einerseits könnte die Erwerbslosigkeit mindestens eines Partners als Chance gesehen werden, einen Kinderwunsch umzusetzen. Auf der anderen Seite können mögliche finanzielle Unsicherheiten, die aus dieser Situation entstehen, divergierende Elternschaftsabsichten begünstigen.

Darüber hinaus zeigen sich Effekte der Beziehungsdauer. Sind Paare drei bis vier Jahre zusammen, weisen sie eine deutlich geringere Chance auf, negative Elternschaftsabsichten zu nennen. Hat die Partnerschaft seit mindestens elf Jahren Bestand, ist für Elternpaare die Chance erhöht, dass beide negative Elternschaftsabsichten angeben. Mit zunehmender Beziehungsdauer haben Elternpaare also häufiger keine Elternschaftsabsicht 
mehr, was wahrscheinlich mit den bereits realisierten Kinderwünschen zusammenhängt (siehe Ergebnisse zur Kinderzahl).

Gehören beide Partner keiner Religionsgemeinschaft an, ist die Chance erhöht, dass beide Partner negative Elternschaftsabsichten äußern. Möglicherweise offenbaren sich hier unterschiedliche Einstellungen zu Kinderzahlen, die über religiöse Werte vermittelt werden. Erwartungsgemäß nimmt auch die Anzahl der Kinder auf Paarebene Einfluss. Im Vergleich zur Referenz (1 Kind) ist die Chance bei Paaren stark erhöht, übereinstimmend negative oder divergierende Elternschaftsabsichten anzugeben, wenn sie schon mindestens zwei Kinder haben.

Für die Kontrollvariablen der Altersdifferenz und der Kinderlosigkeit eines Partners zeigen sich hingegen keine signifikanten Effekte. Schließt man die Personen aus den Analysen aus, die keine Kinder mehr erwarten (Modell 2), fallen einige Effekte schwächer aus bzw. sind nicht mehr signifikant. Dies spricht dafür, dass es sich um eine spezielle Personengruppe handelt.

Im Modell der kinderlosen Paare (Modell 3) weist die Altersdifferenz einen signifikanten Effekt auf, wenn der Mann mindestens vier Jahre älter ist als seine Partnerin. Dann werden seltener übereinstimmend negative Elternschaftsabsichten genannt. Sind kinderlose Frauen jünger als 30 Jahre oder 35 Jahre und älter, ist die Chance erhöht, dass beide Partner negative bzw. divergierende Elternschaftsabsichten äußern. Dies könnte sich über verlängerte Ausbildungszeiten und fehlende berufliche Etablierung, die häufig als Voraussetzung für eine Familiengründung angesehen wird, in dieser Altersgruppe erklären lassen.

Für die Herkunft kinderloser Paare lässt sich festhalten, dass negative Elternschaftsabsichten seltener werden, wenn mindestens ein Partner in Ostdeutschland oder im Ausland geboren wurde. Hier könnte sich der Einfluss von Normen und Werten widerspiegeln. So ist beispielsweise in Ostdeutschland sowie ausländischen Kulturen Kinderlosigkeit wenig verbreitet. Ist nur die Frau bzw. keiner von beiden erwerbstätig, werden häufiger negative bzw. divergierende Elternschaftsabsichten genannt. Ergänzend dazu ist die Chance erhöht, dass beide negative Elternschaftsabsichten angeben, wenn nur der Mann erwerbstätig ist. Die berufliche Etablierung beider Partner scheint eine Vorraussetzung für die geplante Familiengründung Kinderloser zu sein.

Beträgt die Beziehungsdauer bei den kinderlosen Paaren neun bis zehn Jahre, werden seltener negative Elternschaftsabsichten von beiden Partnern geäußert. Mit zunehmender Beziehungsdauer scheinen sich kinderlose Paare in ihren Elternschaftsabsichten anzunähern bzw. eine Umsetzung dieser anzustreben.

Die Zugehörigkeit zu einer Religionsgemeinschaft und die Bildungskonstellation auf Paarebene weisen keine signifikanten Effekte auf. Werden Personen aus den Analysen ausgeschlossen, die keine Kinder mehr erwarten (Modell 4), fallen einige Effekte schwächer bzw. nicht mehr signifikant aus. Dies unterstreicht die Vermutung, dass Personen, die realistisch keine Kinder mehr erwarten, eine besondere Subpopulation abbilden. 


\subsection{Unterscheiden sich Eltern und Kinderlose in ihrer Beziehungszufriedenheit?}

Auch wenn im engeren statistischen Sinn durch die unterschiedlichen Modelle für Eltern und Kinderlose keine Aussage über einen direkten Vergleich beider Gruppen gemacht werden kann, scheint sich kein systematischer Unterschied zwischen Elternpaaren und kinderlosen Paaren anzudeuten. Um dies genauer zu prüfen, wurden Interaktionsterme aus der subjektiven Beziehungszufriedenheit und dem Elternschaftsstatus in einem Modell berechnet, in dem Eltern und Kinderlose zusammen betrachtet werden (Modelle 5 und 6 in Tabelle 4). Die Interaktionen machen deutlich, dass sich Elternpaare und kinderlose Paare in Bezug auf den Einfluss der subjektiven Beziehungszufriedenheit (beide zufrieden bzw. mindestens einer unzufrieden) auf die Elternschaftsabsicht nicht signifikant unterscheiden. Dies gilt für die Personengruppe inklusive und exklusive der Paare, die realistisch kein Kind mehr erwarten. Hypothese 3 zur unterschiedlichen Wirkung der Partnerschaftsqualität auf die Elternschaftsabsicht von Eltern und kinderlosen Paaren muss daher verworfen werden. Eine Interaktion in die Modelle aufzunehmen, bringt somit keinen weiteren Wissenszugewinn im Vergleich zu den Ausgangsmodellen.

Tabelle 4: Ergebnisse der multinomialen Regression zu übereinstimmenden Elternschaftsabsichten (EA) auf Paarebene mit Interaktionstermen zu Beziehungszufriedenheit und Elternschaftsstatus, Eltern und Kinderlose in einem Modell, Relative Risk Ratios (RRR) ausgewiesen

\begin{tabular}{|c|c|c|c|c|c|c|c|c|}
\hline & \multicolumn{4}{|c|}{$\begin{array}{c}\text { Gesamtmodell } \\
\text { Modell } 5 \\
\text { (positive EA (RG)) }\end{array}$} & \multicolumn{4}{|c|}{$\begin{array}{c}\text { ohne Personen mit realistisch } \\
\text { erwarteter Kinderzahl=0 } \\
\text { Modell } 6 \\
\text { (positive EA (RG)) }\end{array}$} \\
\hline & \multicolumn{2}{|c|}{ Negative EA } & \multicolumn{2}{|c|}{ Diverg. EA } & \multicolumn{2}{|c|}{ Negative EA } & \multicolumn{2}{|c|}{ Diverg. EA } \\
\hline & RRR & $\mathbf{p}$ & RRR & $\mathbf{p}$ & RRR & $\mathbf{p}$ & RRR & $\mathbf{p}$ \\
\hline \multicolumn{9}{|c|}{ Beziehungszufriedenheit der Partner } \\
\hline beide (sehr) zufrieden (RG) & 1 & & 1 & & 1 & & 1 & \\
\hline mindestens einer unzufrieden & 1,71 & 0,044 & 1,53 & 0,166 & 1,72 & 0,048 & 1,54 & 0,157 \\
\hline \multicolumn{9}{|l|}{ Elternschaftsstatus } \\
\hline kinderlose Paare & 1 & & 1 & & 1 & & 1 & \\
\hline Elternpaare & 1,13 & 0,609 & 0,84 & 0,563 & 0,79 & 0,395 & 0,81 & 0,490 \\
\hline \multicolumn{9}{|l|}{ Interaktionsterm } \\
\hline $\begin{array}{l}\text { Partnerschaftszufriedenheit* } \mathrm{El} \\
\text { schaftsstatus }\end{array}$ & 1,09 & 0,786 & 1,21 & 0,629 & 1,08 & 0,828 & 1,24 & 0,583 \\
\hline $\mathbf{N}$ & \multicolumn{4}{|c|}{2.126} & \multicolumn{4}{|c|}{1.390} \\
\hline
\end{tabular}

$\mathrm{p}<=0,001$ (höchst signifikant), $\mathrm{p}<=0,01$ (hoch signifikant), $\mathrm{p}<=0,5$ (sign.), $\mathrm{p}<=0,1$ (schwach sign.)

Datenbasis: pairfam 2008/09, Release 2.0, eigene Berechnungen, ungewichtete Daten; kontrolliert für Altersdifferenz, Herkunft, Bildungskonstellation, Erwerbskonstellation, Beziehungsdauer, Religionszugehörigkeit und Kinderlosigkeit auf Paarebene, Alter der Partnerin sowie für fehlende Werte in den Kontrollvariablen, EA = Elternschaftsabsichten, $\mathrm{RG}=$ Referenzgruppe 


\section{Zusammenfassung und Diskussion}

In diesem Beitrag standen die zeitnahen Elternschaftsabsichten von Paaren im Vordergrund. Die Paarperspektive erscheint für die Analyse von Fertilitätsentscheidungen wesentlich, da eine Beziehung für die meisten Personen als Voraussetzung für eine Familiengründung und -erweiterung gilt. Zudem können unter Verwendung der Angaben des Partners gänzlich neue Fragestellungen adressiert werden, die mit Daten, in denen nur individuelle Kinderwünsche enthalten sind, unbeantwortet bleiben müssten, auch wenn dies zum Teil neue methodische Herausforderungen mit sich bringt. Ein solch innovativer Untersuchungsgegenstand ist die Frage, ob die Beziehungszufriedenheit Einfluss auf die Übereinstimmung der Intentionen von Paaren hat. Ausgangslage für die Analysen war die Annahme, dass eine hohe Partnerschaftsqualität einen begünstigenden Einfluss auf übereinstimmend positive Elternschaftsabsichten hat und eine niedrige Partnerschaftsqualität diesen entgegenwirkt. Beziehungen, in denen beide Partner zufrieden sind, sollten einen höheren (austauschtheoretischen) Nutzen für die Beteiligten haben und die gemeinsame Entscheidung für Kinder begünstigen. Die vorliegenden Analysen wurden auf Paarebene, getrennt für Eltern- und kinderlose Paare, durchgeführt.

Ein zentrales Ergebnis des vorliegenden Beitrags ist, dass ein relativ großer Anteil der Paare, unabhängig von ihrem Elternschaftsstatus, übereinstimmend positive bzw. negative Elternschaftsabsichten aufweist. Dies gilt vor allem für Elternpaare (siehe Tabelle 1). Dies entspricht den Ergebnissen von Cavalli und Rosina (2011) sowie Testa et al. (2011), die ähnlich niedrige Prozentsätze für divergierende Elternschaftsabsichten von Elternpaaren finden konnten. ${ }^{5}$ Die kinderlosen Paare in der vorliegenden Stichprobe geben mit 20 Prozent im Vergleich jedoch fast doppelt so häufig an, dass sie voneinander abweichende Intentionen haben. Insofern verdient diese Gruppe in der künftigen Forschung mehr Aufmerksamkeit, um beispielsweise zu klären, ob der fehlende Kinderwunsch des Lebenspartners mit einer dauerhaften Kinderlosigkeit im Zusammenhang steht.

Damit lässt sich die Titelfrage dieses Beitrags wie folgt beantworten: Paare äußern häufiger ein gemeinsames ,ja“ oder „,nein“ als ein „vielleicht“ (divergierende Elternschaftsabsichten). Dabei erscheint vor allem der Elternschaftsstatus relevant, denn kinderlose Paare weisen häufiger divergierende Elternschaftsabsichten auf als Eltern. Einen Erklärungsansatz liefern die möglichen Unsicherheiten, die mit einer Familiengründung einhergehen. Kinderlose sind im Schnitt jünger als Eltern und stehen noch am Anfang ihrer beruflichen und privaten Lebensplanung. Die Umsetzung von Kinderwünschen ist nicht zwingend mit allen angestrebten Lebenswegen vereinbar und wird durch den fehlenden Konsens bei den zeitnahen Elternschaftsabsichten wahrscheinlich erst einmal aufgeschoben. Dies schafft ein Zeitfenster, um sich auszuprobieren.

Darüber hinaus lässt sich festhalten, dass die Beziehungszufriedenheit mit den Elternschaftsabsichten von Kinderlosen und Elternpaaren korreliert. Dabei spielt nicht nur das Ausmaß der Zufriedenheit eine Rolle, sondern auch die möglicherweise unterschiedliche Einschätzung durch die Partner. Es zeigt sich, dass wenn mindestens ein Partner unzufrie-

5 Für den direkten Vergleich eignen sich aus den im Forschungsstand genannten Referenzen lediglich die Daten von Cavalli und Rosina (2011) sowie Testa et al. (2011), da dort ebenfalls eine Aufteilung nach Kinderlosen und Eltern vorgenommen wird. 
den mit der Beziehung ist, häufiger übereinstimmend negative bzw. divergierende Elternschaftsabsichten auf Paarebene beobachtet werden können. Dies bestätigt den austauschtheoretischen Hintergrund dieses Beitrags der besagt, dass eine hohe Beziehungszufriedenheit, welche die Zufriedenheit der Partner mit dem Austausch materieller und immaterieller Ressourcen widerspiegelt, übereinstimmend positive Elternschaftsabsichten auf Paarebene begünstigt. Dies gilt für Eltern und kinderlose Paare gleichermaßen, was für einen elternschaftsstatusunabhängigen Effekt spricht. Neben der Beziehungszufriedenheit nehmen auch andere Lebensbereiche wie die Herkunft oder die Erwerbskonstellation Einfluss auf übereinstimmend positive bzw. negative oder divergierende Elternschaftsabsichten.

Zudem ist anzumerken, dass sich Elternpaare und Kinderlose hinsichtlich der Wirkung der Beziehungszufriedenheit auf die Elternschaftsabsichten nicht signifikant voneinander unterscheiden. Dies gilt mit und ohne Einbeziehung der Personengruppe, die keine Kinder mehr erwartet. Grundsätzlich nimmt diese besondere Subpopulation Einfluss auf die Ergebnisse der Wirkung der Beziehungszufriedenheit auf die Übereinstimmung der Elternschaftsabsichten von Paaren. Schließt man sie von den Analysen aus, zeigt sich, dass der Effekt der Beziehungszufriedenheit für Eltern noch einmal verstärkt wird. Für Kinderlose fällt der Effekt etwas geringer aus. Dennoch stellen die Personen, die keine Kinder mehr erwarten, eine wichtige Untersuchungsgruppe dar. Denn warum sich Paare keine (weiteren) Kinder wünschen, wurde bisher noch unzureichend analysiert.

Abschließend sei noch einmal darauf verwiesen, dass die vorliegenden Ergebnisse auf Querschnittsdaten basieren. Daher können mit den vorliegenden Ergebnissen keine endgültigen Aussagen zu Kausalzusammenhängen zwischen der Partnerschaftsqualität und übereinstimmend positiven Kinderwünschen auf Paarebene getroffen werden. Zwar ist mit der Art der Frageformulierungen eine gewisse zeitliche Distanz zwischen der Partnerschaftsqualität, die sich auf den Befragungszeitpunkt bezieht, und der Elternschaftsabsicht, welche in die Zukunft gerichtet formuliert wird, gewährleistet. Dennoch müssen weitere Paaranalysen im Längsschnitt zur Klärung der Frage beitragen, ob ein gemeinsamer, positiver Kinderwunsch nun tatsächlich aus einer hohen Partnerschaftsqualität resultiert oder ob die Partnerschaftsqualität hoch ist, weil ein gemeinsamer, positiver Kinderwunsch vorliegt (vgl. Pavetic/Stein 2011: 11, 21). Mit der Verwendung von Querschnittsdaten muss zudem die Frage offen bleiben, ob Eltern und kinderlose Paare unterschiedliche Phasen im individuellen Lebensverlauf repräsentieren oder ob es sich um verschiedene Personengruppen handelt. Weitere Erhebungswellen des Beziehungs- und Familienpanels (pairfam) können zu einer Klärung dieser Fragen beitragen.

\section{Danksagung}

Dieses Papier verwendet Daten des Beziehungs- und Familienpanels (pairfam), koordiniert von Josef Brüderl, Johannes Huinink, Bernhard Nauck und Sabine Walper. Pairfam wird als Langzeitprojekt der Deutschen Forschungsgemeinschaft (DFG) gefördert. Mein besonderer Dank gilt Julika Hillmann für ihre Unterstützung und Anmerkungen zu diesem Beitrag. Weiterhin möchte ich den Mitgliedern des Arbeitsbereiches Ökonomische und Soziale Demografie am Max-Planck-Institut für demografische Forschung sowie den anonymen Gutachter(inne)n der Zeitschrift für Familienforschung/Journal of Family Research für wichtige Hinweise und konstruktive Anregungen zu diesem Beitrag danken. 


\section{Literatur}

Arránz Becker, O. (2008): Was hält Partnerschaften zusammen. Psychologische und soziologische Erklärungsansätze zum Erfolg von Paarbeziehungen. Wiesbaden: VS Verlag für Sozialwissenschaften.

Ajzen, I. (1991). The theory of planned behavior. Organizational Behavior and Human Decision Processes, 50, S. 179-211.

Ajzen, I. \& Klobas, J. (2013). Fertility intentions: An approach based on the theory of planned behavior. Demographic Research, 29, 8, S. 203-232.

Becker, G. S. (1974). A theory of social interaction. Journal of Political Economy, 82, 6, S.1063-1093.

Becker, G. S. (1981). A treatise on the family. Cambridge \& London: Harvard University Press.

Borchardt, A. \& Stöbel-Richter, Y. (2004). Die Genese des Kinderwunsches bei Paaren: eine qualitative Studie. Wiesbaden: Bundesinstitut für Bevölkerungsforschung (Materialien zur Bevölkerungswissenschaft, Heft 114)

Bulatao, R. A. (1981). Values and disvalues of children in successive childbearing decisions. Demography, $18,1, \mathrm{~S} .1-25$.

Cavalli, L. \& Rosina, A. (2011). An analysis of reproductive intentions of Italian couples. Population Review, 5, 1, S. 21-29.

Diefenbach, H. (2005). Die Rationalität von Kinderwünschen und reproduktivem Verhalten. Einige Anmerkungen zur konzeptionellen Weiterentwicklung des „Value-of-Children“-Modells. In: Steinbach, A. (Hrsg.), Generatives Verhalten und Generationenbeziehungen. Festschrift für Bernhard Nauck zum 60. Geburtstag. Wiesbaden: VS Verlag für Sozialwissenschaften, S. 111-129.

Eckhard, J. \& Klein, T. (2006). Männer, Kinderwunsch und generatives Verhalten: eine Auswertung des Familiensurvey zu Geschlechterunterschieden in der Motivation zur Elternschaft. Wiesbaden: VS Verlag für Sozialwissenschaften.

Eckhard, J. \& Klein, T. (2007). Die Motivation zur Elternschaft. Unterschiede zwischen Männern und Frauen. In: Konietzka, D. \& Kreyenfeld, M. (Hrsg.), Ein Leben ohne Kinder: Kinderlosigkeit in Deutschland. Wiesbaden: VS Verlag für Sozialwissenschaften, S. 275-294.

Engelhardt, H. (2004). Fertility intentions and preferences: Effects of structural and financial incentives and constraints in Austria. Wien: Vienna Institute of Demography (Vienna Institute of Demography Working Papers 02/2004).

Ette, A. \& Ruckdeschel, K. (2007). Die Oma macht den Unterschied! Der Einfluss institutioneller und informeller Unterstützung für Eltern auf ihre weiteren Kinderwünsche. Zeitschrift für Bevölkerungswissenschaft, 32, 1-2, S. 51-72.

Gloger-Tippelt, G. S. \& Huerkamp, M. (1998). Relationship change at the transition to parenthood and security of infant-mother attachment. International Journal of Behavioral Development, 22, 3, S. 633-655.

Hahlweg, K. \& Baucom, D. H. (2008). Partnerschaft und psychische Störungen. Göttingen: Hogrefe Verlag (Fortschritte der Psychotherapie, Band 34).

Hagewen, K, J. \& Morgan, S. P. (2005). Intended and ideal family size in the United States, 1970-2002. Population and Development Review, 31, 3, S. 507-527.

Hayford, S. R. (2009). The evolution of fertility expectations over the life course. Demography, 46, 4, S. 765-783.

Heiland, F., Prskawetz A. \& Sanderson, W. C. (2008). Are individuals' desired family sizes stable? Evidence from West German panel data. European Journal of Population, 24, S. 129-156.

Hill, P. B. \& Kopp, J. (2002). Familiensoziologie: Grundlagen und theoretische Perspektiven. Wiesbaden: Westdeutscher Verlag.

Höhn, C., Andreas Ette, A. \& Ruckdeschel, K. (2006). Kinderwünsche in Deutschland. Konsequenzen für eine nachhaltige Familienpolitik. Stuttgart: Robert Bosch Stiftung (Hrsg.).

Huinink, J., Brüderl, J. et al. (2011). Panel Analysis of Intimate Relationships and Family Dynamics (pairfam) - Conceptual framework and design. Bremen, Mannheim, Chemnitz \& München: Pairfam (pairfam-Arbeitspapier Nr. 17). 
Huinink, J. \& Konietzka, D. (2007). Familiensoziologie: Eine Einführung. Frankfurt am Main: Campus Verlag.

Jurgan, S., Gloger-Tippelt, G. \& Ruge, K. (1999). Veränderungen der elterlichen Partnerschaft in den ersten 5 Jahren der Elternschaft. In: Reichle, B. \& Werneck, H. (Hrsg.), Übergang zur Elternschaft: Aktuelle Studien zur Bewältigung eines unterschätzten Lebensereignisses. Stuttgart: Ferdinand Enke Verlag, S. 37-51.

Klaus, D. \& Suckow, J. (2005). Der Wert von Kindern und sein langer Schatten. Eine kritische Würdigung der VOC-Forschung. In: Steinbach, A. (Hrsg.), Generatives Verhalten und Generationenbeziehungen. Festschrift für Bernhard Nauck zum 60. Geburtstag. Wiesbaden: VS Verlag für Sozialwissenschaften, S. 85-110.

Klein, T. (1996). Der Altersunterschied zwischen Ehepartnern. Ein neues Analysemodell. Zeitschrift für Soziologie, 25, 5, S. 346-370.

Klein, T. (2003). Die Geburt von Kindern in paarbezogener Perspektive. Zeitschrift für Soziologie, 32, 6, S. 506-527.

Klein, T. \& Eckhard, J. (2008). Partnerschafts- und berufsbezogene Aspekte des Kinderwunsches von Männern und Frauen. In: Feldhaus, M. \& Huinink, J. (Hrsg.), Neuere Entwicklungen in der Beziehungs- und Familienforschung. Vorstudien zum Beziehungs- und Familienentwicklungspanel (PAIRFAM). Würzburg: Ergon Verlag.

Lillard, L. A. \& Waite, L. J. (1993). A joint model of marital childbearing and marital disruption. Demography, 30, 4, S. 653-681.

Miller, W. B. (2010). Fertility desires and intentions: Construct differences and the modeling of fertility outcomes. Paper presented at the conference "From intentions to behaviour: Reproductive decisionmaking in a macro-micro perspective", Vienna Institute of Demography, Vienna (Austria), December 2-3, 2010.

Miller, W. B. \& Pasta, D. J. (1995). Behavioral intentions: Which ones predict fertility behavior in married couples? Journal of Applied Social Psychology, 25, 6, S. 530-555.

Myers, S. M. (1997). Marital uncertainty and childbearing. Social Forces, 75, 4, S. 1271-1298.

Nauck, Bernhard (1989). Individualistische Erklärungsansätze in der Familienforschung: Die rationalchoice-Basis von Familienökonomie, Ressourcen- und Austauschtheorien. In: Nave-Herz, R. \& Marefka, M. (Hrsg.), Handbuch der Familien- und Jugendforschung. Band 1. Neuwied \& Frankfurt am Main: Luchterhand.

Nauck, B. \& Kohlmann, A. (1999). Values of Children - ein Forschungsprogramm zur Erklärung von generativem Verhalten und intergenerativen Beziehungen. In: Busch, F. W., Nauck, B. \& NaveHerz, R. (Hrsg.), Aktuelle Forschungsfelder der Familienwissenschaft. Würzburg: Ergon Verlag.

Nave-Herz, R. (2004). Ehe- und Familiensoziologie. München: Juventa Verlag.

Norton, R. (1983). Measuring marital quality: A critical look at the dependent variable. Journal of Marriage and the Family, 45, 1, S. 141-151.

Pavetic, M. \& Stein, P. (2011). Entscheidungsprozess zur Familiengründung in Partnerschaften. Zeitschrift für Familienforschung/Journal of Family Research, 23, 1, S. 5-23.

Peuckert, R. (1999). Familienformen im sozialen Wandel. Opladen, Leske + Budrich (3., vollständig überarbeitete Auflage).

Reichle, B. \& Werneck, H. (1999). Übergang zur Elternschaft und Partnerschaftsentwicklung: Ein Überblick. In: Reichle, B. \& Werneck, H. (Hrsg.), Übergang zur Elternschaft: Aktuelle Studien zur Bewältigung eines unterschätzten Lebensereignisses. Stuttgart: Ferdinand Enke Verlag, S. 1-16.

Rijken, A. J. \& Liefbroer, A. C. (2009). The influence of partner relationship quality on fertility. European Journal of Population, 25, S. 27-44.

Rijken, A. J. \& Thomson, E. (2011). Partners' relationship quality and childbearing, Social Science Research, 40, S. 485-497.

Rosina, A. \& Testa, M. R. (2009). Couples' first child intentions and disagreement: An analysis of the Italian case. European Journal of Population, 25, S. 487-502.

Schmidt, U. \& Moritz, M.-T. (2009). Familiensoziologie. Bielefeld: transcript Verlag. 
Siffert, A. \& Bodenmann, G. (2010). Entwicklung eines neuen multidimensionalen Fragebogens zur Erfassung der Partnerschaftsqualität. Zeitschrift für Familienforschung/Journal of Family Research, 22, 2, S. 242-255.

Sobel, M. E. \& Arminger, G. (1992). Modeling household fertility decisions: A nonlinear simultaneous probit model. Journal of the American Statistical Association, 87, 417, S. 38-47.

Testa, M. R. (2012). Couple disagreement about short-term fertility desires in Austria: Effects on intentions and contraceptive behaviour. Demographic Research, 26, 3, S. 63-98.

Testa, M. R., Cavalli, L. \& Rosina, A. (2011). Couples' childbearing behaviour in Italy: Which of the partners is leading it? Vienna Yearbook of Population Research, 9, S. 157-178.

Thibaut, J. W. \& Kelley, H. H. (1959). The social psychology of groups. New York: Wiley.

Thomson, E. (2001). Family size preferences. In: Smelser, N. J. \& Baltes, P. B. (Hrsg.), International encyclopedia of the social and behavioral sciences. Oxford: Pergamon Press, S. 5347-5350.

Thomson, E. \& Colella, U. (1992). Cohabitation and marital stability: Quality or commitment? Journal of Marriage and the Family, 54, 2, S. 259-267.

Thomson, E. \& Hoem, J. M. (1998). Couple childbearing plans and births in Sweden. Demography, 35, 3, S. 315-322.

van Peer, C. (2002). Desired and achieved fertility. In: Klijzing, E. \& Corijn, M. (Hrsg.), Dynamics of fertility and partnership in Europe: Insights and lessons from comparative research. Volume II. New York \& Geneva: United Nations, S. 117-142.

Xu, X. (1998). Marital quality revisited: A replication and extension of the JWEB model. Sociological Spectrum, 18, S. 367-382.

Eingereicht am/Submitted on: 30.04 .2012

Angenommen am/Accepted on: 28.08.2013

Anschrift der Autorin/Address of the author:

Anne-Kristin Kuhnt, Dipl.-Demographin, M.Sc.

Max-Planck-Institut für demografische Forschung

Konrad-Zuse-Straße 1

18057 Rostock

Deutschland/Germany

E-Mail:kuhnt@demogr.mpg.de 